\title{
Sobre um caso de paraplegia espasmodica Diagnostico differencial
}

Observação da Clinica Neurologica da Faculdade, feita pelo doutorando João Alves Meira.

Data de entrada: 18-5-1927. Data de observação 15-6-1927. Nome: J. H. S. Cor branca. Estado civil solteiro.

Edade - 23 annos - Profissão - lavrador. Nação - Brasileiro - Naturalidade - Bahia - Pocedencia - Sertãosinho Enfermaria - 2.a M. H. (serviço do Prof. Rubião Meira) - Leito n. ${ }^{\circ} 24$.

\section{ANAMNESE :}

QueıIXa: - Não pode andar. DURAÇão - do inicio da molestia, 7 mezes - da impossibilidade da marcha -5 mezes - HISTORIA DA MOLESTIA ACTUAL - Ha questão mais ou menos de 7 mezes, comę̧ou a sentir uma dormencia nos membros inferiores e uma falta de força mais accentuada no esquerdo. Ia andar e, acontecia muitas vezes tropeçar visto a falta de força na perna esquerda não o ajudar.

No inicio de sua molestia passou 24 horas sem urinar, e, quando, passado esse tempo, conseguiu urinar a sua miç̧ão foi muito dolorosa e pouco mais que gottejante.

Muita vez isso se repetiu até que, com a ingestão de umas capsulas, que não sabe informar o que continham, passou a urinar mais regularmente, embora ainda e sempre com dysuria.

A dormencia e a falta de força foram tratadas como rheumatis mo e nenhum beneficio tirou desse tratamento. Dois mezes após o inicio de sua molestia, marcado pela falta de força da perna esquerda e pela perturbação urinaria a que nos referimos, começou a sentir a dormencia e a falta de força, tambem no membro direito. Até então trabalhava, pois, embora perturbado na marcha que fazia mancando e ás vezes com o auxilio de um bastão, sentia-se apto para o seu serviço.

Após o acommettimento do membro direito não poude mais andar. Sentia as pernas sem jogo, presas, muito rigidas e impossibilitadas de effectuar os movimentos necessarios para caminhar. 
Nem em pé podia-se manter. Tratou-se com injecções de estrychnina (!) sem nenhum resultado - continuando as suas pernas muito rigidas. Conservou-se então no leito. Quinze dias mais tarde começou a sentir forte sensação de queimadura, calor intenso que per. corria todos os 2 membros inferiores. Tinha a impressão que suas vestes estavam ardendo e essa sensação se estendia desde a raiz dos membros até a extremidade distal. Não refere, o doente, á perturbações esphyncterianas outras que as assignaladas. Nada diz quanto á rachialgia, nem á claudicação intermittente. A sua molestia evoluiu sem febre e a perturbação urinaria de começo referida não mais appareceu. Por se sentir impossibilitado de se entregar ao seu trabalho em vista do estado de seu membro inferior que rigido impedia a sua marcha, pelas sensações paresthesicas sentidas e assignaladas e por necessitar de recursos therapeuticos resolveu procurar esse hospital onde deu entrada no dia 18 de Maio deste anno vindo a occupar o leito n. ${ }^{\circ} 24$ da $2 .^{a}$ enfermaria de homens.

\section{INTERROGATORIO SOBRE OS DIFFERENTES APPARELHOS}

Tem cephaléa de vez em quanto, é ligeira e tanto apparece á noite como durante o dia, passando por si mesma.

Ha 4 annos essa cephaléa era acompanhada por diplopia e quando olhava parecia ver "vagalumes" no ar (sic); na mesma occasião não tinha photophobia nem rigidez de nuca.

Não tinha febre. Tudo isso com o tem passou.

Para o ladio da audição todos os informes são negativos. Interrogatorio negativo para as vias areas superiores, e para o apparelho cardio respiratorio.

Apparelho gastro intestinal. Sempre teve e tem bom appetite. Digestão facil. Vomitos e hematemeses - nunca teve. Não assignala gastralgia. Prisão de ventre habitual ha 2 annos. Após o inicio de sua molestia a constipação de ventre se accentuou, passando, 3 á 4 dias, sem evacuar.

Genito-urinario - Nocturia com dysuria. Accorda para urinar e urina pouco e com dor. Tem, muita vez, sensação que precisa evacuar e no entanto o que necessita é urinar. Nada informa quanto á perturbação da erecção nem da ejaculação. Não se refere á impotencia coeundi - Memoria boa. Intelligencia lucida. Psychimo integro.

Antecedentes pessoaes: - Teve as molestias peculiares á infancia todas ellas sem complicações.

Impaludismo ha 3 annos - esteve doente durante 9 mezes Tratou-se, e, depois desse tempo não veio a ter mais accessos. Grippe no anno passado. Nega outras doenças infecciosas. Antecedentes venereos-syphiliticos:

Blenorrhagia ha 4 ou 5 annos da qual se diz curado. 
Nega, terminantemente, todo outro e qualquer antecedente venereo-syphilitico; responde negativamente ás perguntas que se lhe faz á respeito de cancro duro, roseolas e placas mucosas.

Faz uso de bebidas alcoolicas - bebe irregularmente, ora pouco, ora muito, desde muito tempo. Muita vez aconteceu ficar embriagado.

Fumante inveterado. Faz uso de café em abundancia. Toxicos e entorpecentes - não os conhece.

Trabalhava como lavrador, com boa disposição até o aia em que começou a se sentir doente. Come bem, com muito appetite. Dorme mal porque tem constantemente insomnia. O seu peso anterior era 64 kilos. Aos 10 annos soffreu um coice de animal no thorax que lhe acarretou uma deformação do thorax, na face posterior, e da columna vertebral, sem no entanto lhe causar outro incommodo.

ANTECEDENTES HeREDITARIOS: - Sua mãe falleceu ha 13 annos victimada por accidente de parto. De seu pae ha 2 annos que não tem noticias - até então era muito forte e sadio.

Foi seu pae casado 3 vezes sempre com mulheres sadias.

Tem 10 irmãos, todos vivos e sadios. NINGUEM DA SUA FAMILIA SOFFRE DO MESMO MAL QUE O DOENTE APRESENTA OU DE OUTRO COM ELLE PARECIDO. Sua mãe teve um aborto. Nenhum nati-morto. Não sabe informar se as outras esposas de seu pae tiveram abortos ou nati-mortos.

\section{EXAME GERAL}

Aspecto geral sadio (o individuo no leito, com os membros cobertos); facies não carateristica; typo morphologico longelineo; regular desenvolvimento do panniculo adiposo e dos musculos; bom desenvovimento dos ossos.

Decubito indifferente mas para o conseguir recorre ao auxilio dos braços - prefere o decubito dorsal porque lhe é mais natural. Marcha - não pode andar porque as suas pernas não deixam Estação - Não se mantem, pela mesma razãio em pé. Pelle - de coloração normal - secca, apresenta pequenas cicatrizes no abdomem e, na face posterior da coxa esquerda, uma cicatriz recente determinada por quemadura por emplastros collocados sob nodulos determinados por injecção. Mucosas visiveis - normaes. Pellos com desenvolvimento do typo masculino. Unhas sem caracteristicos.

Systema ganglionar: - G. Epitrochleano esquerdo do tamanho de um avellã; do lado direito não se palpa. Inguinaes - ganglios numerosos e de tamanhos varios. Axillares - palpapel á esquerda do tamanho de um grão de ervilha.

Tibialgia e esternalgia - ausentes. 


\section{EXAME ESPECIAL}

Cabeça: - Craneo symetrico, sem exostoses nem pontos dolorosos. Couro cabelludo normal; OLHOS com pupillas iguaes de contornos regulares, reagindo normalmente á luz e á accommodação. Reflexo consensual presente.

Não ha paralysia, nystagmus, nem lacrimejamento.

Diplopia e photophobia ausentes. Conjunctivas coradas normalmente. Orelhas sem tophus nem corrimento. Nariz sem signal de obstrucção, sem deformidade ou corrimento. Seios da face indolores. Bocca - sem ulcerações, pigmentações nem exsudatos. Dentes bons, bem conservados e tratados. Gengivas sadias. Lingua ligeiramente hypotonica, sem tremor, de coloração vermelha intensa e sem saburra. Não se constata ulcerações ou placas mucosas. Amygdalas de tamanho normal, congestas. Pharynge de coloração intensa, sem catarrho. Não ha paralysia. Reflexo pharyngeano presente.

Pescoço: - Thyroide não é palpavel. Não ha thrill, nem puIsações arteriaes ou venosas visiveis; Olliver-Cardarelli-ausente.

Thorax: - O thorax anteriormente é symetrico - claviculas salientes - fossas supra e infra claviculares pouco deprimidas. Angulo de Louis pouco accentuado. Charpy medindo mais ou menos $90^{\circ}$ Costellas bem recobertas; espaços intercostaes normalmente espaçados. Phenomeno de Litten não pesquizado. Typo respiratorio costo-abdominal.

Posteriormente o thorax é asymetrico devido á grande saliencia que faz o hemithorax esquerdo onde o doente recebeu, em pequeno, um coice de animal - o que, aliás, - fora isso, não lhe trouxe maior incommodo. Não ha escapula alatae.

Não se consegue julgar se existe algum desvio da columna porque o doente não fica em pé nem se assenta correctamente no leito. Assume sempre uma attitude viciosa que prejudica o juizo que se poderia fazer, entretanto com uma melhor apreciação constata-se a existencia de um cypho escoliose-dorsal.

Palpação: - O fremito thoraco vocal acha-se conservado no que diz respeito ás suas relações topographicas.

Percussão: - Som claro pulmonar em toda area respiratoria.

Ausculta: - Murmurio vesicular em toda zona pulmonar

Coração: - Choque da ponta no $5 .^{\circ}$ espaço intercostal esquerdo para dentro da linha mamillar. Palpação - Palpa-se com difficuldade o ictus cordis que é fraco e limitado. Não se constata fremito. Percussão - Não praticada. Ausculta. Bulhas com os seus caracteres normaes. Retumbancia da 2. ${ }^{a}$ bulha no foco aortico (signal Rubião Meira). Não ha sopro que denuncie qualquer lesão oro-valvular. 
Aorta: - Não é accessivel atrás da furcula external nem ultrapassa o rebordo do externo á percussão.

Pulso radial: -72 pulsações por minuto. Pulso rythmico, frequente. Paredes arteriaes molles $\mathrm{e}$ facilmente depressiveis. Pressão arteral - Mx12 - Mn7 - (Vaquez-Laubry).

Arterias: - Humeral e temporal - nada de anormal.

Abdomen: - Ligeiramente saliente. Não ha sensação de onda; macicez movel e de flanco ausentes. Não ha sensação dolorosa nem expontanea nem provocada.

Tympanismo á percussão. Figado - Limite superior do figado no $5 .^{\circ}$ espaço intercostal direito ao nivel da linha mamillar. $\mathrm{O}_{i}$ bordo inferior não attinge o rebordo costal. $\mathrm{O}$ figado mede de altura ao nivel da linha mamillar $-12 \mathrm{cms}$. Baço nem palpavel nem percutivel.

Calon sigmoide palpavel na fossa illiaca esquerda sob a forma de um cordão da grossura de um dedo; gosa de mobilidade normal e é indolor. Na fossa illiaca direita palpa-se o caecum. Não conseguimos palpar nem o colon ascendente nem o transverso e descendente assim como o estomago.

Apparelbo genital: - Penis de tamanho normal, sem cicatrizes, ulcerações nem corrimento. Testiculos normaes dolorosas á pressão mais ou menos forte. Bolsa normal.

\section{EXAME DO SYSTEMA NERVOSO}

Estatica: - No exame da estatica verificamos, digno de nota, a presença da cypho-escoliose dorsal já assignalada. O equilibrio na posição vertical é impossivel dado o estado dos membros inferiores do doente. Orientação - Quando praticamos, no nosso doente, as manobras habituaes e classicas que nos devem informar da noção das posições segmentarias obtemos respostas imprecisas, dubias, não nos permittindo tirar conclusões seguras a esse respeito. Podese, entretanto, dizer pelo exame que fizemos, não existir perturbação marcada da noção das attitudes segmentares.

Pela impossibilidade do equilibrio na estação vertical resulta não se poder pesquizar o signal de Romberg. E' integra, no nosso doente, a noção do corpo no espaço.

Motilidade: - a) latente - hypertonia accentuada dos membros inferiores. Nos superiores eutonia. $b$ ) - activa voluntaria: Marcha-o doente não anda; força dynamometrica - não verificada. Paralysia - Constata-se a existencia da paralysia de ambos os membros inferiores (paraplegia ou melhor paraparesia accentuadissima).

Não ha outras perturbações taes como dysmetria, diadococinesia, nem ataxia. Apraxia, agnosia ausentes. Sinergia não verificada. Os movimentos activos são limitadissimos quasi nullos - o 
doente não conseguindo flectir a perna sobre a coxa e essa sobre a bacia. Os joelhos nesse movimento (estando os membros completamente estendidos) não se levantam senão alguns centimetros do plano onde repousam. Para se assentar no leito ou para mudar do decubito dorsal habitual o doente tem que lançar mão dos membros superiores que agarram fortemente a cama. Sem esse auxilio não consegue se erguer no leito, $c$ ) - activa involuntaria: Não ha tremor, nem athetose, nem myoclonia, nem choréa, tiques nem contractura. Espamocidade da musculatura dos membros inferiores.

Não estão presentes os signaes de Kerning nem os phenomenos de Raimisti, Hoover, Strümpell, Babinski, Grasset e Gaussel, Neri, Souques, Klippel e Weil.

d) - Movimentos passivos - presentes e conservados. Articulações livres. Pés ligeiramente cahidos pelo relaxamento dos flexores.

Sensibilidade geral. - Subjectiva; sensações paresthesicas, taes como dormencia, formigamento, sensação de queimadura; dor nos membros inferiores, - objectiva: a) superficial: hyperesthesia cutanea em todo o membro paraparetico, mais accentuada acha-se essa hyperalgesia na face antero externa das pernas e na planta de ambos os pés. Sensibilidade tactil conservada. A sensibilidade thermica difficil de ser pesquisada porque o doente informa mal e com evidente má fé - como que nos querendo ludibriar, d'ahi não se poder tirar conclusão alguma nessa parte do exame. $b$ ) - objectiva profunda - Sensibilidade ossea ao diapasão, não pesquisada. As outras acham-se perfeitas. (Muscular, articular). Sentido estereognostico integro. Sensibilidade especial:

Visão, audição, olfacção e gustação - integras, pelo menos ao exame summario pois não recorremos ao auxilio de especialistas.

Reflectividade: - a) superficial - reflexo cutaneo plantar em extensão ou signal de Babinski presente em ambos os lados.

Reflexo cremasterino presente. Cutaneo abdominaes ausenquer os superiores quer os medios e inferiores.

Refledtividade profunda. - Reflexo patellar exaltado em ambos os lados, com polycinesia e augmento da area reflexogena. Reflexos achilianos exaltados.

Reflexos bicipital, tricipital e radial presentes.

Clonus dos pés e clonus das rotulas, presentes, nitidos e duradouros. Muita vez, clonus expontaneo.

Dos reflexos de automatismo medular, de defeza, reflexos tonicos de postura só encontramos nitido e integral o phenomeno dos encurtadores, o que encontramos de ambos os lados, usando a manobra aconselhada por Foix e Pierre Marie. Todos os outros foram pesquizados com resultado negativo.

Reflexosá luz e á accomodação presentes. Não ha, pois, Argyll Robertson. Reflexo consensual presente. Occulo cardiaco não pesquisado. Reflexo pharyngeano - presente. 
Tropbicidade: - Não ha perturbações trophicas. Ausencia de atrophia quer nos membros superiores quer nos inferiores.

Exame electrico. - Não foi praticado.

Exame mental. - Não ha perturbação da linguagem; Não ha dysarthria nem anarthria nem aphasia.

Noção do logar, do meio e tempo perfeita. Nada de anormal que caracterise a capacidade e nivel mentaes bem como o humor, é encontrado.

Exame psychologico. - Attenção - boa, Memoria não perturbada e associação de ideas como no normal.

Não ha delirios nem alucinaçōes.

Exames de laboratorio.

Wassermann no sangue - negativo.

Urina - sem albumina, nem assucar - não ha sedimentos pathologicos.

Exame do liquido cephalo rachidiano:

A tensão não foi tomada por falta do apparelho de Claude, na occasião. O liquido é transparente como agua de rocha. Exame cytologico 3 lymphocytos por millimetro cubico.

Reacção de Pandy - negativa - Reacção de Wassermann positiva fracamente $(\div)$. As reacções de Nonne - Apelt, do benjoin colloidal (G. Laroche, Guillain, Lechelle) e a reacção de Weichbrodt bem como a dosagem da albumina, embora pedidas, não foram feitas por falta de material, pois o que foi enviado ao laboratorio Central era insufficiente para tal fim $(6 \mathrm{cms} .3)$. Não quizemos fazer uma segunda puncção lombar porque a primeira determinou, dor, sensação vertiginosa ao lado de outros incommodos, todos, aliás, fugazes.

Exame radiologico da columna vertebral:

Revelou a existencia de uma cypho-escoliose dorsal, já constatada pelo exame physico, com achatamento lateral dos corpos vertebraes das $7 .^{\mathrm{a}}, 8 .^{\mathrm{a}}, 9 \mathrm{a}^{\mathrm{a}}$ e $10 .^{\mathrm{a}}$ dorsaes.

Fizemos repetir mais de uma vez o exame radiologico sempre com o mesmo resultado. Resumo da observação com os signaes physicos positivos.

Doente ha 7 mezes; inicio com difficuldade da marcha por fraqueza do membro inferior esquerdo.

Fatigabilidade da marcha que ás vezes se fazia tropega. Perturbação urinaria de inicio com retenção de urina por 24 horas. Micção penosa e difficil. Constipação de ventre habitual. Dois mezes depois acommettimento do membro inferior direito. Paralysia de ambos os membros inferiores. Não anda e não fica o doente em pé. Perturbações paresthesicas (dormencia, formigamento, sensação de queimadura). Nega antecedentes syphiliticos. Exame: Retumbancia da $2 .^{\mathrm{a}}$ bulha do do foco aortico. Ganglio epithocheano 
esquerdo palpavel bem como os inguinaes e axillares. Cypho escoliose dorsal.

Reflexos: - Patellar exaltados. Achilianos exaltados. Babinsky de ambos os lados, Reflexo dos encurtadores (automatismo) presente. Clonus as rotulas. Clonus dos pés. Clonus expontaneo. Hypertonia dos membros inferiores.

Paralysia dos membros. Hyperalgesia cutanea da perna e da planta dos pés. Não ha perturbação da sensibilidade profunda. Argyll - Roberston - ausente; Romberg - impossivel pesquisar .

Diagnostico. - Paraplegia espasmodica por myelite syphilitica dorso-lombar num portador de cypho-escoliose dorsal traumatica.

Evolução: - O doente que já se submetteu ao tratamento antiluetico tem obtido melhoria embora ligeira.

Deve-se confiar no resultado do mesmo tanto no que diz respeito ao estacionamento do processo morbido quanto ao desapparecimento da paraplegia. Contudo o prognostico é reservado. (quod valitudinem).

Tratamento: - Anti-luetico mixto, isto é, arsenical e bismuthico ou arsenico-mercurial. Convem tambem o emprego do iodeto de sodio em injecções endovenosas.

\section{I S CUSS Ã O}

Pela leitura da observação e pelo resultado dos exames procedidos no nosso doente verifica-se tratar desde logo de um caso de paraplegia medular espasmodica, forma "grabataire" typo em extensão. Caracterisando a paraplegia espasmodica encontramos a impotencia motora dos membros inferiores (paraparesia) acompanhada de uma rigidez espatica com contractura dos mesmos e signaes de hyperreflexia (Babinsky - clonus dos pés e rotulas, exaltação dos reflexos patellar e achiliano - reflexo dos encurtadores (Foix Pierre Marie) e associada a um estado de hypertonia. Caracterisando a forma "grabataire" está presente o facto de se achar o individuo immobilisado ao leito. E, finalmente, marcando o typo em extensão encontra-e a attitude pelo doente assumida no leito, com adducção forçada das coxas e os pés com equinismo, embora ligeiro, e em adducção. Quanto ao dizer-se paraplegia medular. A simples leitura da observação nos informa satisfactoria e sufficientemente para a localisação do processo morbido dispensando maiores commentarios.

Que é então, o nosso doente, portador de uma paraplegia espasmodica não ha que duvidar. Mas a que quadro etiologico se enquadra esse estado morbido no nosso doente?

E' o que passamos á discutir.

Num caso como o nosso as hypotheses dignas de estudo são as seguintes: paraplegia espasmodica: $a$ ) na esclerose em placas, $b$ ) na 
syringomyelia, $d$ ) na esclerose lateral amyotrophica, $d$ ) - nas escleroses combinadas; $e$ ) - paraplegia das creanças e adolescentes; $f$ ) - na hemato-myelia expontanea; $g$ ) - nas compressões medulares; $b)$ - na syphilis medular.

Vejamos agora, mais minuciosamente, de qual dessas paraplegias é o nosso doente portador. Estudemos pois os caracteristicos de cada uma das formas suppostas para approximal-as ou afastal-as do nosso caso.

A) - A paraplegia espasmodica na esclerose em placas consoante ao facto das placas de esclerose se disseminarem pelo nevraxe atacando frequentemente o cerebello é via de regra acompanhada de outros signaes não apresentados, aliás pelo nosso doente. Assim é que por não se encontrar o tremor intencional, o nystagmus, os signaes cerebellosos, a palavra explodida ou escandida nem as pupillas descoradas (estado do fundo de olho) pode-se afastar o diagnostico de paraplegia-espasmodica na esclerose em placas. Além disso o nosso doente apresentou perturbações esphyncterianas que são excepcionaes na esclerose em placas.

B) - Na syringomyelia, a paraplegia, no caso espasmodica, é raramente crural, o mais das vezes costuma situar-se nos membros superiores, onde se desenvolvem as atrophias musculares e consequentes deformações proprias de tal especie morbida. Nada disso se passando no nosso caso e principalmente a ausencia da chamada dissociação syringomyelica afasta completamente a syringomyelia da scena morbida do nosso doente.

C) - Na esclerose lateral amyotrophica a paraplegia espasmodica mais ou menos accentuada é obrigatoriamente acompanhada por atrophia muscular com os caracteristicos das atrophias myelopathicas, tremor fibrillar, reacção de degeneração, etc. A ausencia de atrophia, no nosso paciente, afasta plenamente a hypothese da esclerose lateral amyotrophica bem como uma atrophia muscular progressiva do typo Aran-Ducheune com o que se pode confundir clinicamente o mal de Charcot antes se installar os signaes espasticos.

$D)$ - Afasta-se, no nossa caso, a possibilidade de se tratar de paraplegia espasmodica nas escleroses combinadas por dois principaes factores.

$1 .^{\circ}$ ) - Ausencia dos signaes da serie tabetica (ataxia, signal de Romberg, abolição do reflexo achiliano, o rotuliano achando-se exaltado ou abolição dos reflexos com signal de Babinsky (por exemplo).

$2 .^{\circ}$ ) - Ausencia do factor etiologico das escleroses combinadas. (pellagra, lathyrismo, anemia perniciosa, etc.).

$E)$ - Por se tratar no nosso caso, de um individuo moço presta-se, no estabelecimento do dignostico differencial á discussão das paraplegias da infancia e dos adolescentes. 
Essas, podem ser divididas em congenitas, paraplegia da infancia e paraplegia da adolescencia.

Nas paraplegias congenitas - forma espasmodica, encontramos como exemplo typico a syndrome de Little

A doença de Litte por ser, via de regra, o resultado de um parto laborioso, (forceps) encontrando-se, tambem, nos prematuros, após toxi-infecção da mãe na gravidez e por isso manifestando-se desde muito cedo - $5 .^{\circ}$ ou $6 .^{\circ}$ mez ou quando a creança faz os seus primeiros passos - é afastada porque como vimos da leitura da observação, a doença, no nosso caso, se manifestou na idade adulta. A simples leitura da observação com effeito, exclue a hypothese da syndrome de Little, quer na sua forma espinal (Degerine e Madame Long - Landry) quer na sua forma cerebral.

Entre as paraplegias da adolescencia só nos depara a paraplegia espinal espasmodica familiar (Strümpell) que é afastada pelo facto de no nosso caso, como frizamos na observação, não revestir, a paraplegia espasmodica, o caracter familiar.

$F)$ - A hypothese de uma hematomyelia expontanea é facilmente afastada porque, pode-se dizer, sem temor, faltam ao caso presente todos os caracteristicos da hermato-myelia expontanea. Assim é que esse processo morbido se installa em certos predispostos, fazendo seguida a um esforço ou apparecendo no curso das molestias hemorrhagicas.

Nada disso se observa no nosso caso, demais a paraplegia espasmodica na hemato myelia expontanea é secundada á forma flacida que sobrevem com inicio apoplectiforme, ás vezes, com perda do conhecimento, dores rachidianas, perturbações esphyneterianas e dissociação sgringomyelica.

A morte, via de regra, sobrevem em alguns dias por escaras de decubitus e quando ha sobrevivencia, nota-se amyotrophia progressiva em certos territorios paralysados ao nivel da lesão, passando, como já dissemos, então a paraplegia de flacida á espasmodica.

Pelo que acima escrevemos e confrontando com o quadro morbido apresentado pelo nosso doente, vemos que nada ha que sirva de traço de união - d'ahi o excluir-se facilmente a paraplegia espasmodica sobrevindo na hematomyelia expontanea.

G) - A constatação da existencia, no nosso observado, de uma cypho-escoliose dorsal nos obriga a um exame cuidadoso das compressões medulares que podem determinar o quadro de uma paraplegia espastica.

Entretanto, desde já, podemos dizer que a cypho-escoliose dorsal é o resultado do traumatismo (coice de animal) soffrido pelo nosso doente na idade de 10 annos. Demais o exame radiologico repetido, nos obriga a afastar essa hypothese (compressão) porquanto nada mais encontrou que um achatamento lateral das $7 .^{\mathrm{a}}, 8 .^{\mathrm{a}}, 9 .^{\mathrm{a}}$ e $10 .^{\mathrm{a}}$ vertebras dorsaes. 
Tambem clinicamente, podemos excluir da scena morbida do nosso doente as compressões medulares a saber: mal de Pott (tuberculose vertebral), cancer vertebral, meningite espinhal chronica, hemorrhagia meningea espinhal e tumores da medula.

Assim é que, o mal de Pott á primeira vista, consoante a deformação thoraxica e o desvio da columna vertebral encontradas constituisse um diagnostico de muita probabilidade, foi afastado não só radiologicamente (pedra de toque) mas mesmo clinicamente pela ausencia, no nosso caso, de dores com caracter pseudo nevralgico, do typo radicular. Falta, ainda no nosso caso, a dor á pressão e á percussão vertebraes. Demais a inexistencia da syndrome de Froin, associada aos factores assignalados reforçam o nosso juizo afastando a hypothese de paraplegia espasmodica por mal de Pott.

$\mathrm{O}$ cancer vertebral é facilmente excluido, quer pela idade do doente, quer pela falta de tumor primitivo (o cancer vertebral, via de regra, sendo secundario) e sobretudo pela auencia de um dos seus mais nitidos caracteristicos - a dor "atroz" Ainda o exame radiologico afasta semelhante eventualidade.

Igualmente a ausencia das dores revestindo o caracter radicular exclue o diagnostico de tumor das meninges bem como o de meningite espinhal chronica. O diagnostico de hemorrhagia meningeana espinal é excluido não só pela ausencia das dores do typo radicular e da rachialgia mas tambem porque o liquido rachidiano na hemorrhagia meningeana espinal é sanguinolento.

Os tumores da medula são de diagnostico muito difficil e são casos raros. O diagnostico simplifica-se quando se encontra no liquido cephalo rachidiano as cellulas neoplasicas. Comtudo pelo facto de sua evolução ser rapida e por apresentar perturbação da sensibilidade, muitas vezes, do typo syringomyelico pode-se afastar essa occurencia (tumor da medula) como agente responsavel da paraplegia no nosso caso.

A prova do lipiodol de Sicard, - que seria de grande valia no diagnostico da compressão medular, ao exame pelo Raio $\mathrm{X}$, não poude ser praticada.

Uma vez passada em revista as causas de compressão medular capazes de explicar a paraplegia espasmodica de que é portador o nosso doente, somos levados a dizer, não existir relação de causa e effeito entre a cypho-escoliose dorsal e a paraplegia nelle presentes. Parece-nos que, quando muito, o traumatismo pelo doente soffrido e do qual resultou o referido desvio da columna, não tenha servido senão para a localisação do processo morbido que elle apresenta, isto é, como adeante veremos, da syphilis medular. (myelite syphilitica).

H) - E' na syphilis, antes de tudo, que devemos pensar, consoante os ensinamentos classicos divulgados, quando nos achamos em presença de um paraplegico.

Esse conceito torna-se mais rigoroso quando deante de nós se acha um caso de paraplegia cuja etiologia não é evidente. A pes- 
quiza cuidadosa dos estigmas da syphilis se impoe bem como a puncção lombar. O exame do liquido cephalo rachidiano, em taes casos, nos dá a chave do dignostico e porisso a puncção lombar se impõe.

Analysemos, embora perfunctoriamente, as formas que pode revestir a syphilis medular procurando identificar o nosso caso entre as estudadas.

Entre as formas mais communs devemos estudar a claudicação intermittente da medula devida a um espasmo das arterias medulares tomadas de arterite syphilitica. Nella a paraplegia sobrevem após a marcha e cede após alguns minutos de repouso. Ella precede, via de regra, a paraplegia syphilitica confirmada. A ausencia das paradas bruscas durante a marcha, na historia da doença do nosso paciente, nos leva a afastar semelhante hypothese. A hypothese que em seguida devemos estudar é o de se tratar de um caso de paraplegia espinal espastica syphilitica descripta por Erb. Tem uma evolução muito lenta e inicia-se como uma veradeira claudicação intermittente da medula. Nunca apresenta no inicio phenomenos meningeos. A rigidez muscular dos membros inferiores se installa progressiva e gradualmente sem que a força muscular seja diminuida. Ha nella, um caracter importante, e é a existencia mais de uma contractura que de uma paralysia. O entrave da marcha corre por conta dessa contractura. Essa é bem evidenciada quando o individuo estando com os joelhos approximados, procura-se afastar um do outro; consegue-se isso até uma certa extensão, depois a contractura impede o afastamento. No nosso doente essa manobra é nitidamente negativa conseguindo-se um afastamento total dos joelhos anteriormente aproximados. A manobra inversa tambem resultou negativa. Não ha, nessa forma, nem amyotrophia nem perturbações da sensibilidade fora alguns formigamentos. A essas perturbações vem se ajuntar perturbações dos esphyncteres e necessidade imperiosa de urinar.

No nosso caso não se deve pensar se trate de um caso de paraplegia espinal espastica de Erb por dois factores de ordem clinica a falta do prodomo habitual - claudicação intermittente e sobretudo pela evolução. Com effeito, na paraplegia de Erb como assignalamos a evolução é muito lenta occorrendo, ás vezes, annos para o estabelecimento da impotencia motora. No nosso caso tal não se deu pois não foi lenta, antes mais ou menos brusca (perna esquerda anteriormente) e não foi gradual e progressiva a installação da rigidez. Convem notar entretanto que o nosso caso apresenta semelhança com a paraplegia de Erb no tocante aos signaes de espasmocidade encontrados bem como nas perturbações esphynterianas constatadas.

Excluidas a claudicação intermittente e a paraplegia de Erb devemos pensar, ainda tratando da syphilis medular, na myelite trasversa.

Essa é facilmente excluida quer porque o seu inicio reveste a forma aguda com paraplegia flacida acompanhada de perturbações 
esphyncterianas e da sensibilidade subjectiva e objectiva quer porque a espasmocidade nella encontrada é sempre secundaria.

A inexistencia do caracter agudo, da flaxidez primitiva e sobretudo pela ausencia de perturbaçỗes accentuadas da sensibilidade nos leva a afastar a hypothese de myelite aguda transversa syphilitica.

Das formas raras de paraplegia occorrendo na espondylite syphilitica (mal de Pott syphilitico), na syndrome de Brown Sequard, na syndrome de pseudo-esclerose em placas, na syndrome pseudo tabetica por predominancia da lesão sobre os feixes posteriores, na syndrome pseudo-syringomyelica e na syndrome amyotrophica, não fazemos senão assignar porque o exame procedido no paciente e a sua historia morbida não nos permitte encarar semelhantes eventualidades.

Resta-nos, discutindo ainda a hypothese da syphilis medular, considerar a myelite syphilitica.

Já por exclusão, já pelo quadro symptomatico apresentado pelo nosso doente e pelo exame do liquido cephalo rachidiano podemos affirmar seja o nosso observado portador de uma paraplegia espasmodica por myelite syphilitica.

Com effeito. Uma vez analysadas todas as causas capazes de determinar a paraplegia espasmodica e todas ellas excluidas vemos que só nos resta a myelite syphilitica, o que aliás, explica perfeitamente todos os symptomas apresentados pelo nosso doente e já relatados na nossa observação, ou sejam: paraparesia accentuada dos membros inferiores, com hypertonia, contractura e hyperreflexia, com pequenas perturbações paresthesicas - (formigamento, sensação de calor) insignificante perturbação da sensibilidade (hyperalgesia) e perturbaçōes esphyncterianas, datando desde o inicio da molestia (dysuria, retenção de urina, constipação de ventre).

Ao lado dos factos clinicos temos os dados do laboratorio e assim, o exame do liquido cephalo rachidiano, constatando uma lymphocytose, embora ligeira (3 lymphocytos por mm3) e Reacção de Wassermann positiva $(\div)$ corrobora a nossa asserção, affirmandose tratar de paraplegia espasmodica por myelite syphilitica. Embora o paciente não relate o accidente inicial da syphilis - nelle encontramos estigmas da lues taes como: ganglios infarctados, cephala e retumbancia da 2. ${ }^{\text {a }}$ bulha aortica, e attestando ainda a natureza syphilitica do processo encontramos o facto da melhoria observada com a administração do tratamento antiluetico.

Quanto á sede, ou melhor, á altura da medula onde se assestou o processo morbido - quer nos parecer seja na medula dorso-lombar - não só por ser ahi a sede habitual da myelite syphilitica como tambem por causa dos signaes de localisação que o nosso doente apresenta.

Finalmente, para terminar, podemos em vista do exposto estabelecer o diagnostico seguinte: paraplegia espasmodica por myelite syphilitica, dorso lombar num portador de cypho escoliose dorsal de causa traumatica. 


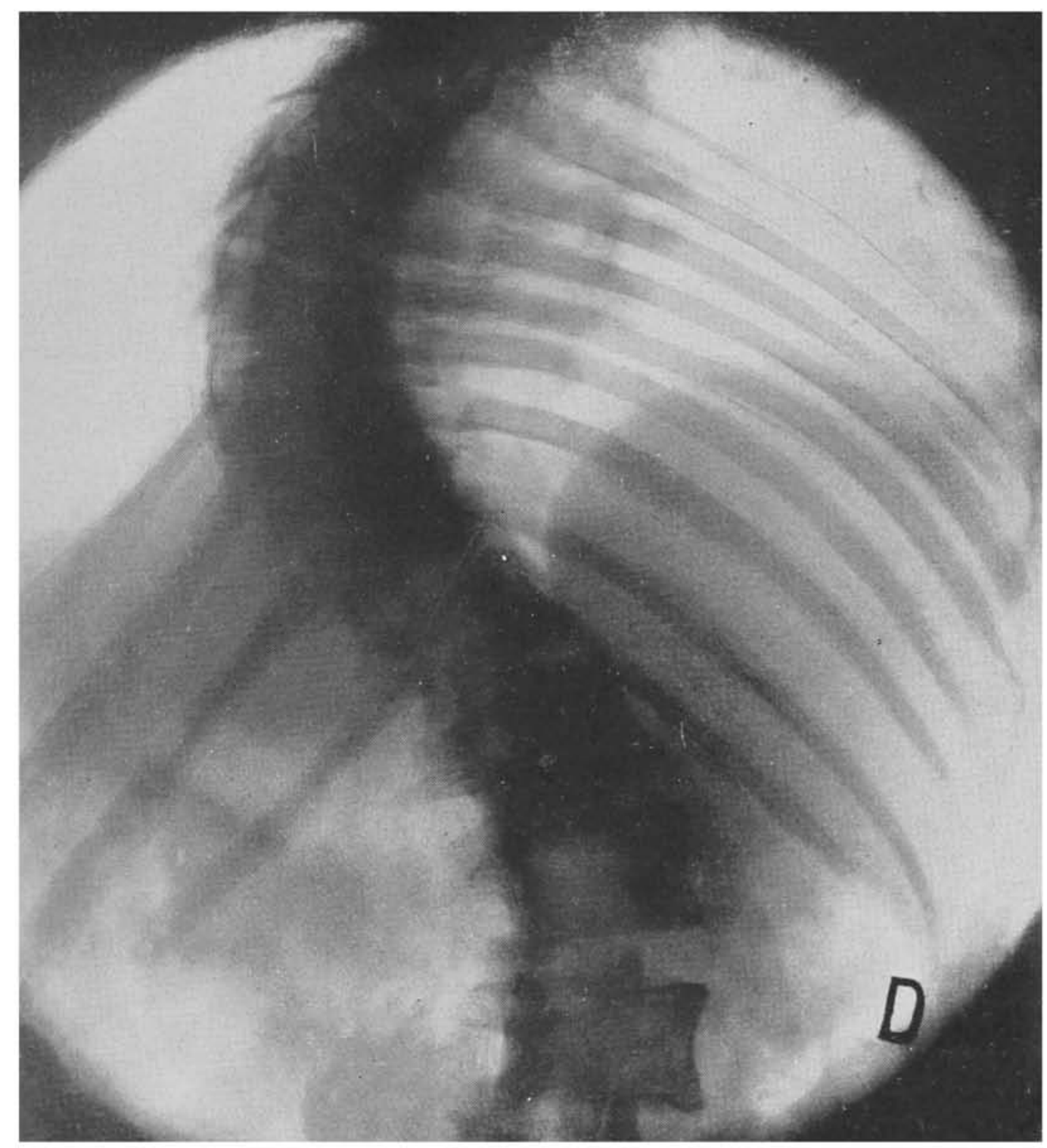

RADIOGRAPHIA DO DOENTE OBSERVADO PELO DOUTORANDO Joĩo Alves Meira. 